\title{
A Framework on A Computer Assisted and Systematic Methodology for Detection of Chronic Lower Back Pain using Artificial Intelligence and Computer Graphics Technologies.
}

\author{
Ala S. Al Kafri ${ }^{1}$, Sud Sudirman ${ }^{1}$, Abir J. Hussain ${ }^{1}$, Paul Fergus ${ }^{1}$, Dhiya Al- \\ Jumeily ${ }^{1}$, Mohammed Al-Jumaily ${ }^{2}$ and Haya Al-Askar ${ }^{3}$ \\ ${ }^{1}$ Faculty of Engineering and Technology, Liverpool John Moores University, Byrom Street, \\ Liverpool, L3 3AF, United Kingdom (UK) \\ ${ }^{2}$ Consultant Neurosurgeon and Spine Surgeon, Dr Sulaiman Al Habib Hospital \\ Dubai Healthcare City, United Arab Emirates (UAE) \\ ${ }^{3}$ College of Computer Engineering and Science \\ Sattam Bin Abdulaziz University, Kingdom of Saudi Arabia (KSA) \\ a.s.alkafri@2015.ljmu.ac.uk; \{s.sudirman, a.hussain, p.fergus, d.aljumeily\}@1jmu.ac.uk; \\ maljumaily@yahoo.fr; sun_2258@hotmail.com
}

\begin{abstract}
Back pain is one of the major musculoskeletal pain problems that can affect many people and is considered as one of the main causes of disability all over the world. Lower back pain, which is the most common type of back pain, is estimated to affect at least $60 \%$ to $80 \%$ of the adult population in the United Kingdom at some time in their lives. Some of those patients develop a more serious condition namely Chronic Lower Back Pain in which physicians must carry out a more involved diagnostic procedure to determine its cause. In most cases, this procedure involves a long and laborious task by the physicians to visually identify abnormalities from the patient's Magnetic Resonance Images. Limited technological advances have been made in the past decades to support this process. This paper presents a comprehensive literature review on these technological advances and presents a framework of a methodology for diagnosing and predicting Chronic Lower Back Pain. This framework will combine current state-of-the-art computing technologies including those in the area of artificial intelligence, physics modelling, and computer graphics, and is argued to be able to improve the diagnosis process.
\end{abstract}

Keywords: Computer Aided/Assisted Diagnosis, Chronic Lower Back Pain, Artificial Intelligence, Physics Modelling, Computer Graphics.

\section{Introduction}

Back pain is one of the major musculoskeletal pain problems which affected many people and it is considered as one of the main causes of disability all over the world [1]. The Pain Community Centre [2] indicated that in the United Kingdom (UK), 2.5 million people have back pain every day of the year. The survey also found that back problems are the leading cause of disability with nearly 119 million days per year lost. The survey also found that one in eight unemployed people give back pain as the reason for unemployment. Statistically, an individual who has been off sick with back pain for a month has a $20 \%$ chance to still being off work a year later [3]. The 
percentage of people who return to see their general practitioner (GP) with back pain within 3 months is more than $29 \%$ [4].

There are two types of back pain, upper and lower ones. Lower back pain is more common than the former and is estimated to affect at least $60 \%$ to $80 \%$ of the adult population in the UK at some time in their lives. While most of them will have resolution of their back pain with simple measures such as using simple analgesia and exercise, a small proportion of them develop a more chronic condition [5][6]. Lumbar spine is the lower back area in the spinal column which contains five vertebrae labelled L1 to L5 [7][8]. Figure 1 describes the lumbar spine and its parts which are the area affected by Chronic Lower Back Pain (CLBP) [9]. Magnetic Resonance Image (MRI) is mainly used to diagnose patients with CLBP or those with symptoms consistent with radiculopathy or spinal column stenosis [10]. Physicians perform the diagnosis normally by studying the MR images through visual inspection of the data. CLBP can be caused by a number of factors including fractures, lumbar disc degeneration, lumbar disc herniation, or infection in the nerve roots. If they suspect disc herniation as a possible cause of the pain, they would utilize axial view of the MRI to help form their decision [11]. In the case of vertebrae infection or fracture, MRI is also the best choice for diagnosis because it allows displaying the full infected area including the bone marrow to differentiate it from more serious cases such as crushed vertebrae [12].

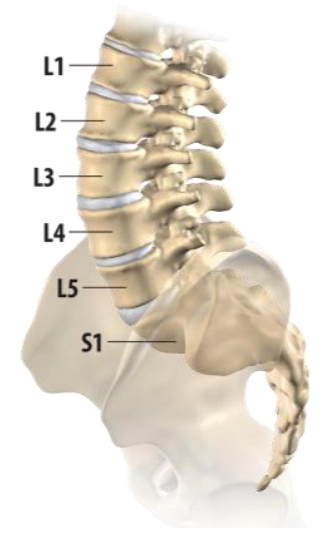

Figure 1. Lumbar Spine that contain the vertebrae from L1 to L5 [13].

Visual observation and analysis of MR images could take up much of a physician time and effort. Moreover, it can increase the probability of misdiagnosis. As a result, physicians would opt to use a Computer Aided Diagnosis (CAD) to help this task. There are a number of CAD systems that can be used for various clinical purposes ranging from a CAD system for detecting colonic polyp and breast cancer in mammography, to another for detecting prostate cancer using MR images [11]. Despite the availability of these systems, physicians still have to overcome a number of technical challenges due to the wide range of imaging characteristics and resolutions [14] as well as due to the limitation of the algorithms employed to highlight areas of interest.

On the opposite end, there are also some progresses in the rehabilitation mechanism of CLBP patients. For example, a lower back pain rehabilitation system is 
proposed in [16] using a wireless sensor technology which helps the patients and physiotherapists carry out the rehabilitation exercises. In addition to the problem of diagnosing the cause of CLBP and rehabilitation of the patients, there is also the issue of prevention. The importance of a reliable prevention mechanism was highlighted in [15] which stated that an accurate means of identifying patients at high risk for chronic disabling pain could lead to more cost-effective care. Furthermore, it can be argued that CLBP is suffered by patients who have history of untreated non-chronic LBP. Therefore, it is imperative that there should be a computer aided system in place to help physicians in their tasks in identifying potential problems that might occur in the future based on existing physiology of the lumbar spine and the patient's characteristics. There has been limited progress in this regard, including one by Neubert et. al [17] who claimed that 3-dimensional MR images have the potential to help physicians to detect and monitor the spine disorder at an early stage. Consequently, we argue that this is one of the most promising areas of research in which computer technologies can play significant part in solving the problem. A number of research works to further the technology in this regard have been made. Ahn [18] developed an interactive computerised simulation of a virtual model of human cervical spine which incorporates physics based modelling and implemented using a physics engine library. Physics engine libraries are traditionally used to develop gaming, robotics, or flight simulations but more recently they are used by researchers for medical purposes [19]. Furthermore, our initial review of the literature reveals that there are some progress in the modelling of the lumbar spine as a $3 \mathrm{D}$ computer model as well as mathematical/physics model [20]-[22]. It is believed that these advances in physics modelling coupled with computer technologies can help solve the problem of future prediction of CLBP. This initial review of the various techniques to detect flaws in lumbar spine and 3D modelling of lumbar spine had highlighted the significance of identifying and understanding a problem space that associated with computer assisted and systematic methodology. We have identified two main research issues associated with this and are proposing two solutions that address them in this paper. These solutions will improve the speed and accuracy of the physicians' and radiologists' tasks in diagnosing and managing CLBP patients. The remainder of this paper is organised as follows. Section 2 presents the literature review of existing techniques that helps diagnosis and management of CLBP. The framework of the proposed system is presented in Section 3. Section 4 contains the discussion and analysis and Section 5 presents the paper's conclusion.

\section{Review of existing techniques in diagnosing and management of CLBP}

There are two main techniques that help in diagnosing and managing CLBP that will be explained in this section.

\subsection{Lumbar disc herniation detection using Computer Vision and Artificial}

\section{Intelligence techniques}

Clinical studies have indicated that morphological characteristics of lumbar discs and their signal intensity on a patient's MRI image have close relationship to the clinical outcome [23]. To this end, computer vision and artificial intelligence algorithms can be utilised to exploit these facts by analysing the MR images, calculating appropriate image features (or feature descriptors), and classifying them to decide if any particular regions in the image belong to problematic areas. Image 
features can be considered as a set of important information derived from an image or a subset of an image that can uniquely describe the image contents. This information is extremely important in computer vision as it can be used to label or mark specific locations of the image and can be used in comparing various images. There are two types of image features namely global and local features [24]. Local features are computed at different locations in the image using only small support area of around the location point. As such, local features describe only the image in the context of that small subset and nothing else. That means even when the other parts of the image undergo changes, as long as the support area remains the same, local features would more likely not be affected. This is one of the strong points of local features over global features because they are robust to occlusion. Examples of local features are corners, edges, and texture descriptors. On the other hand, global features are derived from the entire image that resulted in their ability in generalising the entire image into one single feature vector. One example of global features is image code, which is a compressed form of the image using an appropriate coding technique that preserves the high level information of the image contents. Alternatively, global features could be constructed from a collection of local features such as shape descriptors, contours descriptors, texture descriptors, etc. Image analysis and comparison are performed by means of classifying its features. This is done by comparing the features from the test image in question with those from training data. A brute force approach for comparing two sets of image features would compare every feature in one set to every feature in the other and keeping track of the "best so far" match. This results in a heavy computational complexity in the order of $\mathrm{O}\left(\mathrm{N}^{2}\right)$ where $\mathrm{N}$ is the number of feature in each image. A number of algorithms have been proposed to improve the computational complexity, including the popular kd-tree technique [25]. This technique uses exact nearest neighbour search and works very well for low dimensional data but quickly loses its effectiveness as dimensionality increases. The popularity of the kd-tree technique has seen a number of derivatives that further improve the algorithm including [26], [27]. The success of a more recent matching technique called Fast Library for Approximate Nearest Neighbour (FLANN) [28] is another example how the computational complexity of image feature comparison can be further reduced to allow near real-time execution.

The uniqueness of each proposed algorithm in this category often lies in the choice of features and matching algorithms as well as novel application of existing approaches to new or untested problems. This research reviews a number of algorithms that are proposed to identify regions in the MRI that are responsible for CLBP that explained below.

Jiang et. al. [28] proposed a visualization and quantitative analysis framework using image segmentation technique to derive six features that are extracted from patients MR images, which were found to have close relationship with Lumbar Disc Herniation score. The six features include the distribution of the protruded disc, the ratio between the protruded part and the dural sacs, and its relative signal intensity. Alomari et. al. [11], [29] proposed a probabilistic model for automatic herniation detection that incorporates appearance and shape features of the lumbar intervertebral discs. The technique models the shape of the disc using both the T1-weighted and T2weighted co-registered sagittal views for building a 2 dimensional (2D) feature image. The disc shape feature is modelled using Active Shape Model algorithm while the appearance is modelled using the normalized pixel intensity. These feature-pairs are then classified using Gibbs-based classifier. The paper reported that $91 \%$ accuracy is achieved in detecting the herniation. A vertebrae detection and labelling algorithm of lumbar MR images is proposed in [14]. The paper firstly converts the 2D MR images 
to $3 \mathrm{D}$ before using them as an input to the detection algorithm. This detection algorithm is a combination of two detectors namely Deformable Part Model (DPM) [30] and inference using dynamic programming on chain [31]. After the spines were detected in the 3D images, a graphical model of the spine layout is built and the bounding box for all vertebrae in are labelled. The algorithm is evaluated on a set of 291 lumbar spine test images with variable number of vertebrae visible and is reported to achieve $84.1 \%$ and $86.9 \%$ correct identification rate for overall vertebrae and lumbar vertebrae respectively. A computational method to diagnose Lumbar Spinal Stenosis (LSS) from the patient's Magnetic Resonance Myelography (MRM) and MRI is proposed in [32]. LSS is a medical condition in which the spinal canal narrows and compresses the spine. In this paper, an image segmentation process is first carried out as a pre-processing step to identify the affected dural sac area in the input images. It then produces the relevant image features based on the inter and intra context information of the segments and use them to detect the presence of LSS [32]. Detection of problematic areas in medical images is not the only application of computer vision and AI in medicine. One evidence for this can be seen in [33]. In the paper, an image processing algorithm is used, not for detection, but to improve the clarity and quality of 3D MRI and computed tomography (CT) images so that they can be viewed without using a disparity device.

\subsection{Three-dimensional geometrical and physics modelling of lumbar spine for future prediction of CLBP}

Three-dimensional surface modelling of lumbar spine has been carried out and widely published in the literature. A number of physics models of the lumbar spine have also been developed [34]. However, little of these have been used to help physicians in future prediction of CLBP. This section describes existing related techniques and technologies in this area.

Starting with the type of input data used to generate the 3D model, a study that compares the quality of 3D-surface model generated from both CT scan and MRI is proposed [20]. The research interestingly concluded that CT scan is better than MRI scan in producing adequate surface registration for image segmentation and generation of a 3D-surface model [20]. Most geometrical and dynamic physics modelling of lumbar spine in the literature are carried out using Finite Element Modelling (FEM). One of the earliest techniques that uses FEM is detailed in [22] and [35]. A more recent technique to create 3D geometrical and mechanical model of lumbar spine with FEM is proposed by Nabhani and Wake [36] which modelled the L4 and L5 vertebrae. The paper reports large stress concentrations in the superior and inferior facet region and on the central surfaces of the vertebral body and in the cortical shell of the vertebrae. The software package used to reconstruct the vertebrae model is I-DEAS Master Series. Noailly et. al. also used FEM to model the L3, L4 and L5 vertebrae [37]. The paper, however, offers an inconclusive finding about model validation through comparison of computed global behaviours with experimental results. A number of researches studied the effect of body movement and the application of external pressure on the generated lumbar model. Feipel et. al. studied the kinematic behaviour of the lumbar spine during walking including the effect of walking speed on the lumbar motion (translation, rotation, and bending) patterns [38]. The study concluded that walking velocity affects the range of the lumbar motion but not the sagittal plane motion. Another study by Papadakis [39] shows that there is a statistically significant difference between gait variability in a group of people with lumbar spinal stenosis and a healthy group of individuals. 
Receiver Operating Characteristic (ROC) is used in this study to measure the method value and to find the cut-off value. In addition, to finding the condition in the day of measurement the Oswestry Low Back Pain was used [40]. The effect of external pressure on the shape of lumbar spine is studied in [41]. The paper concludes that a posterior-to-anterior (PA) force which is applied during MRI scan at a single lumbar spinous process causes motion of the entire lumbar region. The findings of these works suggest that there are many factors that can determine the shape of the lumbar spine at any one time and they may affect both the resulting 3D geometry model as well as physics model of the spine. With the advancement in computer graphics and computer game technology, realistic simulation of real life objects ranging from racing cars kinematics to projectiles trajectory and to character movements is becoming a reality. It is therefore sensible to consider these new technologies for more serious applications such as computer aided diagnosis. A study on the appropriateness of using extensible physics engines for medical simulation purposes is given in [19]. A review and survey of recent techniques which use game engines in simulating clinical training is given in [42].

\section{Discussion and Analysis}

Based upon the review of the literature it can be concluded that there are significant gaps that need to be bridged between the relevant computing technologies (such as image processing, artificial intelligence, computer vision, and computer graphics and physics simulation) and their application as an aid tool in the diagnosis and management of CLBP patients. The aim of this research is to develop a computer assisted and systematic methodology for detection and prediction of potential sources of chronic lower back pain using these technologies. Therefore, we hypothesise that the bridging of these gaps, by employing relevant state-of-the-art computing technologies in computer aided system for diagnosis and management of CLBP patients, would improve the efficiency and accuracy of the medical process to diagnose and manage CLBP patients. Previous researchers [5] [43] have highlighted the importance of identifying and understanding the problem area that are related to the medical decision assisted systems. Moreover, the mechanism of artificial intelligence and computer graphics technologies which could be applied in the lower back pain detection and modelling process is another factor which needs to be identified carefully. After extensively reviewing existing research work, we found two important issues that need to be addressed. First, how we can use current advances in computer technologies to help physicians diagnose the cause of CLBP. Second, how we can use current advances in computer technologies to help physicians predict future occurrence of CLBP in their patients.

In this research we are proposing a framework for a novel methodology that utilises state-of-the-art computing technologies, which can be used as a computer aided diagnosis tool, to help physicians in their efforts to diagnose and manage CLBP cases. The methodology consists from two parts: The first part is a lumbar disc herniation detection using computer vision and artificial intelligence (AI) techniques. In this case, the system takes MRI of CLBP patient's lumbar spines as inputs and produces highlights of the lumbar disc if herniation is detected. The development of this part would include 1) the development of novel image features suitable for differentiating herniated and normal discs from their image appearances and 2) finding a suitable and best state-of-the-art Artificial Intelligence technique for classifying the features by analysing and comparing their performances. The second 
part is a 3D geometrical and physics modelling of lumbar spine for analysing the source of CLBP. The model would take into account the patient's characteristics such as height, weight, gender, and age as well as the current state of his/her lumbar spine as derived from the patient's MR images. The model will be used to provide dynamic and interactive 3D visual representation of the patient's lumbar spine to the physician and can simulate the positions in which pain is generated. In addition, the same approach could be used to predict the progression of the degenerative process of the disc.

\section{Framework of the Proposed System}

The proposed system will have two functionalities. Firstly, the system will be used to detect lumbar disc herniation using computer vision and artificial intelligence techniques. In this functionality, the patient's contrast weighted MR images is segmented to remove any irrelevant parts from the image and keep only the disc, vertebrae and spinal cord canal. The system then calculates the disc height [44], the distance between adjacent vertebrae, the distance between disc and spinal cord[45], and the feature descriptors for the disc shape [43]. The system also requires additional inputs such as the patient's age, gender, height and weight. These inputs are used to determine the expected values for the disc height, the distance between adjacent vertebrae, the distance between disc and thecal sac, and the feature descriptors for the disc shape, when abnormalities do not occur. The system then applies a knowledgebased or artificial intelligence algorithm to compare the two sets (the calculated set and the determined set) to decide whether disc herniation occurred. This process is illustrated in Figure 2.

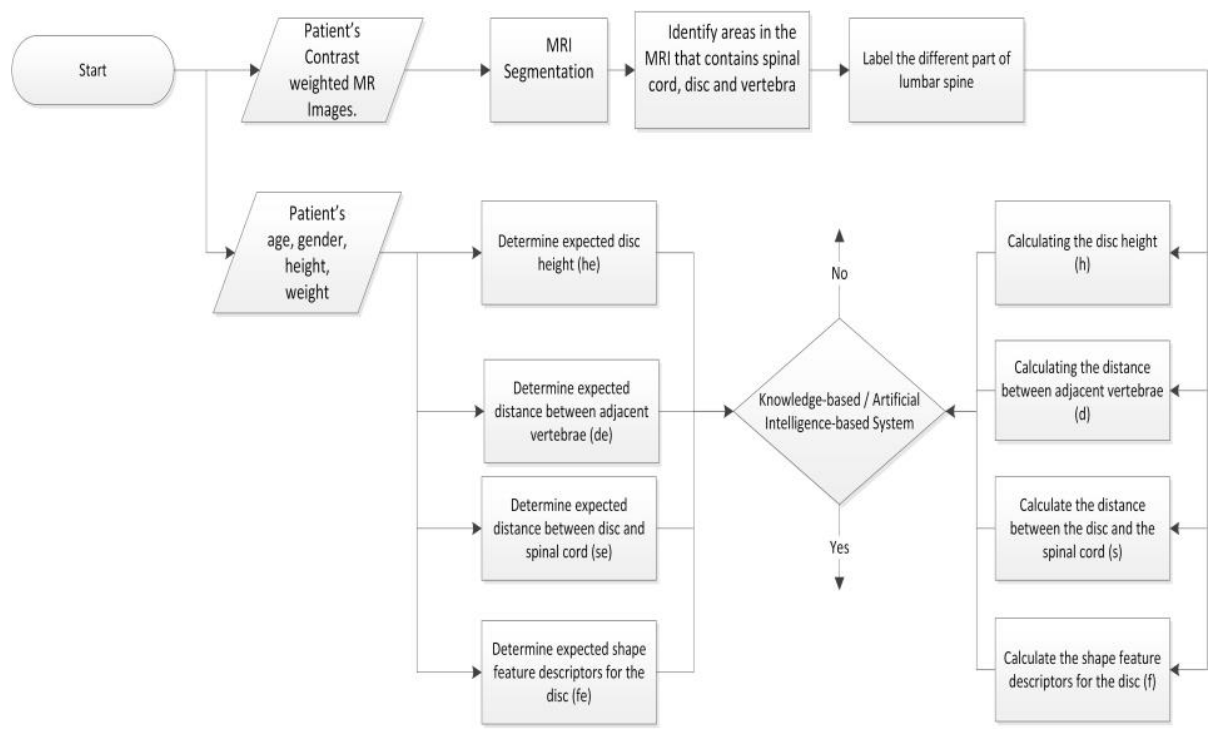

Fig. 2. Steps for Disc Herniation Detection System

There are a number of algorithms that could be used in the knowledge-based/artificial intelligence system. Our approach is to experiment with a number of classifiers and 
image feature descriptors and perform the training and classification process using combinations of them. The best pair of classifier and feature descriptors will be chosen based on their accuracy (high true positive and false negative rates as well as low false positive and true negative rates). To illustrate the training and classification process, we use one of the most popular and widely used classifiers namely the Haar Cascade Classifier [46]. The Haar Cascade is very popular in the Computer Vision research community because it is significantly faster than other similar approach while also providing a very good accuracy. The technique has also been implemented as an open source in OpenCV. Haar Cascade classifier can be trained using thousands of sample images and utilises the sliding window technique to locally compute the feature descriptors. The training will use contrast weighted MR images as inputs as well as labelled affected region that have been done manually. Contrast weighted images are used to emphasis different types of tissues within the same MR images. This process is further illustrated in Figure 3.

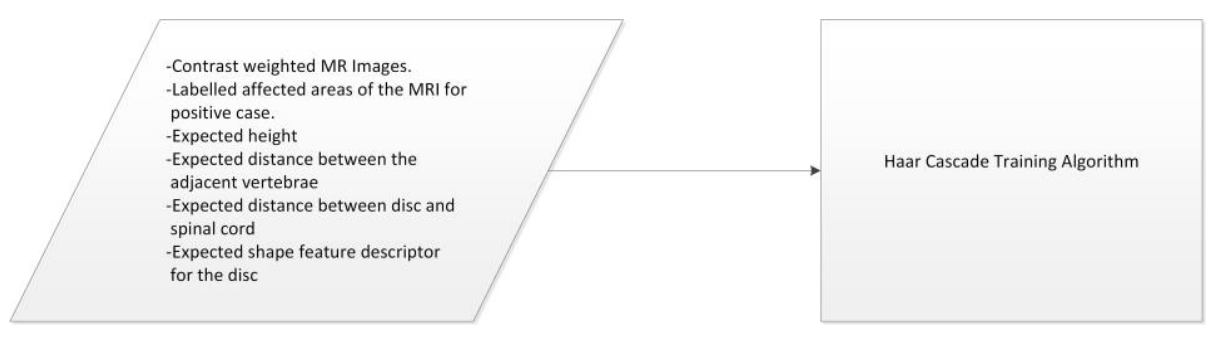

Fig. 3. System Training

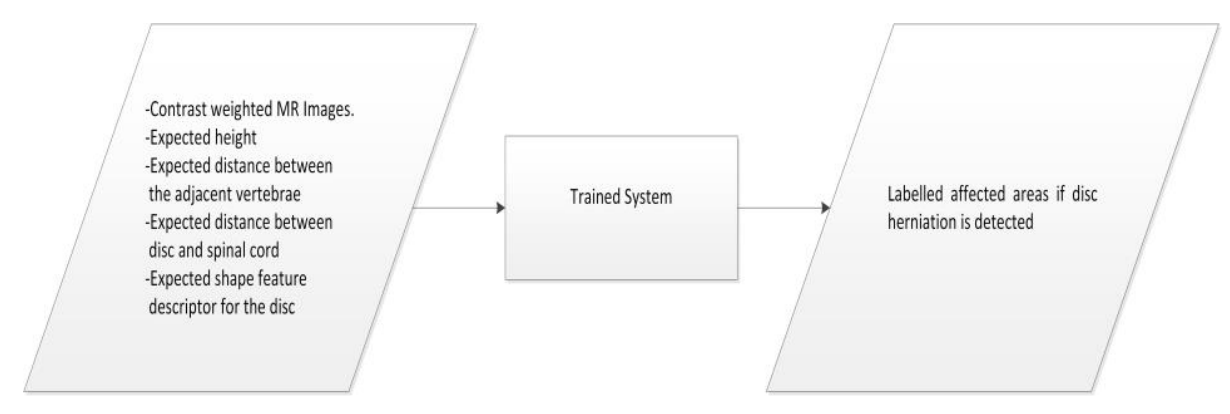

Fig. 4. System Testing

The trained system will then be able to produce labelled images of the affected areas if disc herniation is detected in the input MR images, as shown in Figure 4. For the second functionality, the system will be used to give the physician the ability to predict the future occurrence of disc herniation. This functionality uses the same first step as the first one that is the segmentation of the patient's contrast weighted MR images to remove irrelevant parts. A 3D model of the patient's lumbar spine is then developed using the segmented MR images. A physics model is then attached to this 3D model to govern the kinematics of the lumbar spine. Similar to the first functionality, the system also takes patient's age, gender, height, and weight as the second set of input. This set of input is then used to adjust the parameters of the physics model to adapt it to the patient's characteristics. This will then be used for 
either the visualisation of the lumbar spine and prediction of future occurrence of disc herniation. This process is illustrated in Figure 5.

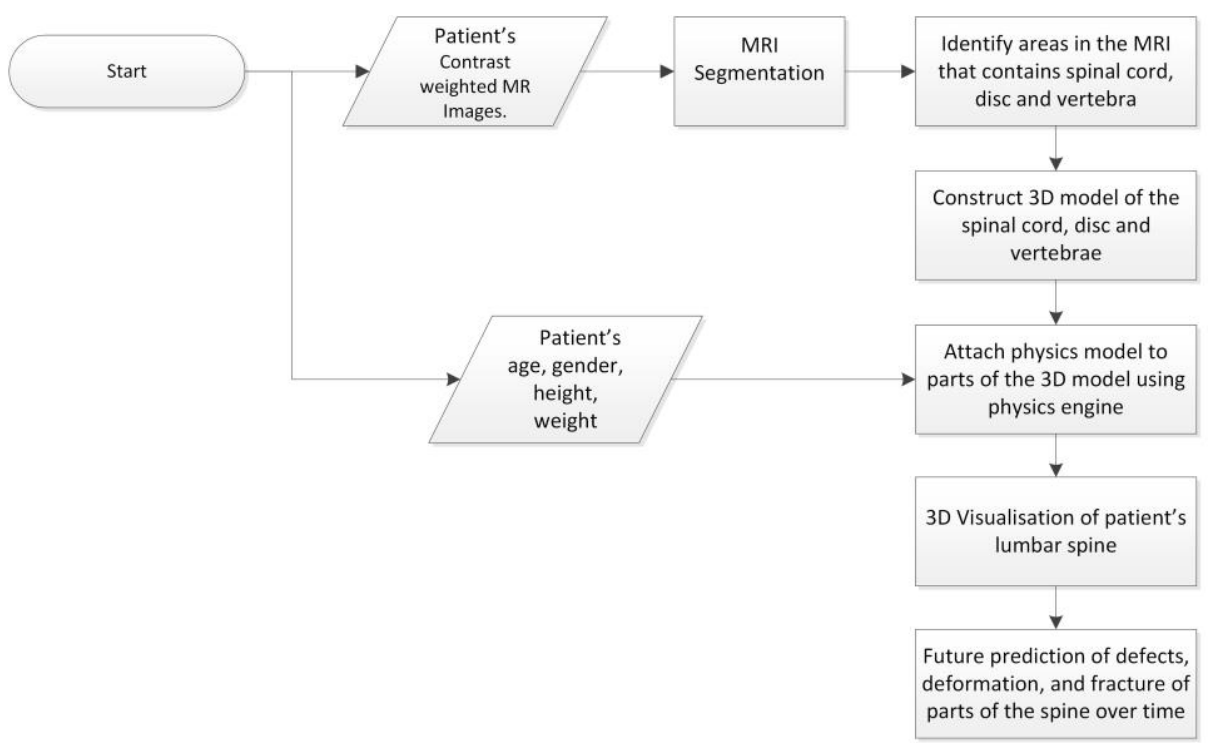

Fig. 5 Steps for Lumbar Spine Visualisation and Prediction System

\section{Conclusion}

The progress in image processing, computer vision, artificial intelligence, computer graphics and physics simulation is moving rapidly in the past decade. However, they have not been utilized in any significant way to improve Computer Aided Diagnostic technique in particular the way Chronic Lower Back Pain cases are diagnosed and managed. Most physicians still relying on a long and laborious task to visually identify abnormalities from the patient's Magnetic Resonance Images. Furthermore, currently there is no existing solution to assist physicians to understand how patients' physiological characteristics, posture and position affect the way pain at lower back spine is generated. This paper proposed a framework for a novel methodology that utilises state-of-the-art computing technologies, which can be used as a computer aided diagnosis tool, to help physicians in their efforts to diagnose and manage CLBP cases. The methodology consists from two parts namely, a) lumbar disc herniation detection using computer vision and artificial intelligence (AI) techniques, and b) 3D geometrical and physics modelling of lumbar spine for visualising lumbar spine and prediction of future occurrence of disc herniation. This proposed system will be able to solve some of the existing problems with the current Chronic Lower Back Pain diagnosis and management procedures.

\section{References}

[1] K. McCamey and P. Evans, "Low back pain.," Prim. Care, vol. 34, no. 1, pp. 71-82, 2007.

[2] "Pain Community Centre." [Online]. Available: http://www.paincommunitycentre.org/article/low-back-pain-problem\#ref6.

[3] G. Waddell, The Back Pain Revolution, 2nd ed. Churchill Livingstone 
(Elsevier), 2004.

[4] P. R. Croft, G. J. Macfarlane, A. C. Papageorgiou, E. Thomas, and A. J. Silman, "Outcome of low back pain in general practice: a prospective study," Br. Med. J., vol. 316, no. 7141, p. 1356, 1998.

[5] G. Waddell and A. K. Burton, "Occupational health guidelines for the management of low back pain at work: Evidence review," Occup. Med. (Chic. Ill)., vol. 51, no. 2, pp. 124-135, 2001.

[6] K. Burton and N. Kendall, "Musculoskeletal Disorders," Bmj, vol. 348, no. feb21 2, p. bmj.g1076-bmj.g1076, 2014.

[7] R. M. Ellis, "Back pain.," BMJ, vol. 310, no. 6989, p. 1220, 1995.

[8] P. Raj, H. Nolte, and M. Stanton-Hicks, "Anatomy of the Spine," Illus. Man. Reg. ..., pp. 1-5, 1988.

[9] P. P. Proximal, P. P. Supplement, C. M. Powers, L. a Bolgla, M. J. Callaghan, N. Collins, and F. T. Sheehan, "Patellofemoral Pain: Proximal, Distal, and Local Factors, 2nd International Research Retreat," J. Orthop. Sports Phys. Ther., vol. 42, no. 6, pp. 1-55, 2012.

[10] R. Methods and B. Practices, "Appropriateness of Care : Use of MRI in the Investigation of Patient Low Back Pain Executive Summary," pp. 1-29.

[11] A. Raja'S, J. J. Corso, V. Chaudhary, and G. Dhillon, "Automatic diagnosis of lumbar disc herniation with shape and appearance features from MRI," in SPIE Medical Imaging, 2010, p. 76241A-76241A.

[12] A. L. David, "9 Spine," Imaging for students, no. D, pp. 187-206, 2012.

[13] "The Healthy Spine | Spinal Simplicity." [Online]. Available: http://www.spinalsimplicity.com/the-healthy-spine/. [Accessed: 29-Mar2016].

[14] M. Lootus, T. Kadir, and A. Zisserman, "Vertebrae Detection and Labelling in Lumbar MR Images," Lect. Notes Comput. Vis. Biomech., vol. 17, no. i.

[15] J. A. Turner, S. M. Shortreed, K. W. Saunders, L. Leresche, J. A. Berlin, and M. Von Korff, "Optimizing prediction of back pain outcomes.," Pain, vol. 154, no. 8, pp. 1391-401, Aug. 2013.

[16] W.-C. Su, S.-C. Yeh, S.-H. Lee, and H.-C. Huang, "A virtual reality lowerback pain rehabilitation approach: System design and user acceptance analysis," in Lecture Notes in Computer Science (including subseries Lecture Notes in Artificial Intelligence and Lecture Notes in Bioinformatics), vol. 9177, 2015, pp. 374-382.

[17] A. Neubert, J. Fripp, C. Engstrom, R. Schwarz, L. Lauer, O. Salvado, and S. Crozier, "Automated detection, 3D segmentation and analysis of high resolution spine MR images using statistical shape models," Phys. Med. Biol., vol. 57, no. 24, p. 8357, 2012.

[18] H. S. Ahn, "A virtual model of the human cervical spine for physics-based simulation and applications," The University of Memphis, 2005.

[19] S. Nourian, X. Shen, and N. D. Georganas, "Role of extensible physics engine in surgery simulations," in Haptic Audio Visual Environments and their Applications, 2005. IEEE International Workshop on, 2005, p. 6 pp.-.

[20] C. L. Hoad, A. L. Martel, R. Kerslake, and M. Grevitt, “A 3D MRI sequence for computer assisted surgery of the lumbar spine," Phys. Med. Biol., vol. 46, no. 8, p. N213, 2001.

[21] S. T. Morais, "Development of a biomechanical spine model for dynamic analysis," Universidade do Minho, 2011.

[22] A. Shirazi-Adl, A. M. Ahmed, and S. C. Shrivastava, "A finite element study of a lumbar motion segment subjected to pure sagittal plane moments," $J$. 
Biomech., vol. 19, no. 4, pp. 331-350, 1986.

[23] R. S. Alomari, J. J. Corso, V. Chaudhary, and G. Dhillon, "Lumbar Spine Disc Herniation Diagnosis with a Joint Shape Model," Clin. Appl. Spine Imaging, vol. 17, pp. 87-98, 2014.

[24] D. A. Lisin, M. A. Mattar, M. B. Blaschko, M. C. Benfield, and E. G. Learned-miller, "Combining Local and Global Image Features for Object Class Recognition," CVPR Work., 2005.

[25] J. H. Freidman, J. L. Bentley, and R. A. Finkel, "An algorithm for finding best matches in logarithmic expected time," ACM Trans. Math. Softw., vol. 3, no. 3, pp. 209-226, 1977.

[26] S. Arya, D. M. Mount, N. S. Netanyahu, R. Silverman, and A. Y. Wu, “An optimal algorithm for approximate nearest neighbor searching in fixed dimensions," Proc. 5th ACM-SIAM Symp. Discret. Algorithms, vol. 1, no. 212, pp. 573-582, 1994.

[27] J. S. Beis and D. G. Lowe, "Shape indexing using approximate nearestneighbour search in high-dimensional spaces," Proc. IEEE Comput. Soc. Conf. Comput. Vis. Pattern Recognit., pp. 1000-1006, 1997.

[28] M. Muja and D. G. Lowe, "Scalable nearest neighbour methods for high dimensional data," IEEE Trans. Pattern Anal. Mach. Intell., vol. 36, no. 11, pp. 1-14, 2014.

[29] R. S. Alomari, J. J. Corso, V. Chaudhary, and G. Dhillon, "Lumbar spine disc herniation diagnosis with a joint shape model," in Computational Methods and Clinical Applications for Spine Imaging, Springer, 2014, pp. 87-98.

[30] P. F. Felzenszwalb, R. B. Girshick, D. McAllester, and D. Ramanan, "Object detection with discriminatively trained part-based models," Pattern Anal. Mach. Intell. IEEE Trans., vol. 32, no. 9, pp. 1627-1645, 2010.

[31] D. P. H. Pedro F Felzenszwalb, "Pictorial Structures for Object Recognition," 2004.

[32] J. Koh, R. S. Alomari, V. Chaudhary, and G. Dhillon, "Lumbar Spinal Stenosis CAD from ClinicalMRMand MRI Based on Inter-and Intra-Context Features with a Two-Level Classifie," vol. 7963, pp. 796304-796304-8, 2011.

[33] J. Kamogawa and O. Kato, "Virtual Anatomy of Spinal Disorders by 3-D MRI / CT Fusion Imaging,” no. Table 1, 2010.

[34] K. Huynh, I. Gibson, and Z. Gao, "Development of a Detailed Human Spine Model with Haptic Interface," Cdn.Intechweb.Org, pp. 165-195, 2012.

[35] F. Lavaste, W. Skalli, S. Robin, R. Roy-Camille, and C. Mazel, "Threedimensional geometrical and mechanical modelling of the lumbar spine," $J$. Biomech., vol. 25, no. 10, pp. 1153-1164, 1992.

[36] F. Nabhani and M. Wake, "Computer modelling and stress analysis of the lumbar spine,” J. Mater. Process. Technol., vol. 127, no. 1, pp. 40-47, 2002.

[37] J. Noailly, H.-J. Wilke, J. A. Planell, and D. Lacroix, "How does the geometry affect the internal biomechanics of a lumbar spine bi-segment finite element model? Consequences on the validation process," J. Biomech., vol. 40, no. 11, pp. 2414-2425, 2007.

[38] V. Feipel, T. De Mesmaeker, P. Klein, and M. Rooze, "Three-dimensional kinematics of the lumbar spine during treadmill walking at different speeds," Eur. spine J., vol. 10, no. 1, pp. 16-22, 2001.

[39] N. C. Papadakis, D. G. Christakis, G. N. Tzagarakis, G. I. Chlouverakis, N. a Kampanis, K. N. Stergiopoulos, and P. G. Katonis, "Gait variability measurements in lumbar spinal stenosis patients: part A. Comparison with 
healthy subjects.," Physiol. Meas., vol. 30, no. 11, pp. 1171-1186, 2009.

[40] N. C. Papadakis, D. G. Christakis, G. N. Tzagarakis, G. I. Chlouverakis, N. A. Kampanis, K. N. Stergiopoulos, and P. G. Katonis, "Gait variability measurements in lumbar spinal stenosis patients: part A. Comparison with healthy subjects," Physiol. Meas., vol. 30, no. 11, p. 1171, 2009.

[41] K. Kulig, R. F. Landel, and C. M. Powers, "Assessment of Lumbar Spine Kinematics Using Dynamic MRI: A Proposed Mechanism of Sagittal Plane Motion Induced by Manual Posterior-to-Anterior Mobilization," J. Orthop. Sport. Phys. Ther., vol. 34, no. 2, pp. 57-64, 2004.

[42] S. Marks, J. Windsor, and B. Wünsche, "Evaluation of game engines for simulated clinical training," in New Zealand Computer Science Research Student Conference (NZCSRSC) 2008, 2008.

[43] R. S. Alomari, J. J. Corso, V. Chaudhary, and G. Dhillon, "Automatic Diagnosis of Lumbar Disc Herniation with Shape and Appearance Features from MRI," Prog. Biomed. Opt. imaging, vol. 11, p. 76241A-76241A, 2010.

[44] S. Ghosh, R. S. Alomari, V. Chaudhary, and G. Dhillon, "Computer-aided diagnosis for lumbar mri using heterogeneous classifiers," Proc. - Int. Symp. Biomed. Imaging, no. MARCH, pp. 1179-1182, 2011.

[45] J. Jordan, K. Konstantinou, and J. O'Dowd, "Herniated lumbar disc.," BMJ Clin. Evid., vol. 2009, 2009.

[46] P. Viola and M. Jones, "Rapid object detection using a boosted cascade of simple features," Proc. 2001 IEEE Comput. Soc. Conf. Comput. Vis. Pattern Recognition. CVPR 2001, vol. 1, no. C, pp. 511-518, 2001. 